\title{
Intervenciones para prevenir la anemia ferropénica del niño prematuro. Controversias
}

\author{
Interventions to prevent iron deficiency anemia in premature children. \\ Controversies
}

\author{
Ana María Menéndez ${ }^{1,2}$, Carmen Vecchiarelli ${ }^{3}$, María Luz Pita Martín de Portela ${ }^{2,4}$ \\ Recibido: 1 de marzo 2018. Aceptado para publicación: 20 de marzo 2018 \\ https://doi.org/10.35454/rncm.v1n1.078
}

\section{Resumen}

El tercer trimestre de gestación es el período de mayor acreción de macro y micronutrientes. Los recién nacidos prematuros presentan un peso inferior a los nacidos a término y deberían lograr un ritmo de crecimiento posnatal que les permita alcanzar la composición de un feto normal de la misma edad posconcepcional. Para ello, se debe instaurar nutrición temprana que aporte cantidades de nutrientes varias veces superiores a la de los recién nacidos de término, que evitará complicaciones durante el período hospitalario. El déficit nutricional es difícil de compensar, pese a la administración precoz de nutrición parenteral y enteral con leche humana fortificada. El hierro es esencial en el organismo y su depleción genera anemia ferropénica, frecuente en esta población asociada a alteraciones del neurodesarrollo. Existen medidas de prevención como el clampeo tardío del cordón y el control de las extracciones de sangre durante la internación. Sin embargo, surgen algunas controversias, en cuanto a las dosis de hierro, la duración del tratamiento, la vía de administración, los criterios transfusionales, la posible asociación con enterocolitis y el tratamiento con eritropoietina.

Se debe evitar el exceso de hierro debido a los riesgos en el aumento de incidencia de la retinopatía del prematuro.

En la práctica actual, el pediatra se enfrenta con la disyuntiva de elegir entre diversas estrategias para administrar cantidades de hierro que eviten la anemia ferropénica y los problemas derivados de su exceso, para evitar costos económicos y sociales a corto y largo plazo, optimizando el desarrollo y la salud en la infancia, adolescencia y adultez.

Palabras clave: hierro, recién nacido prematuro, nutrición, anemia ferropénica.

1 Cátedra de Farmacia Hospitalaria y Clínica. Carrera de Farmacia, Universidad de Belgrano, Ciudad Autónoma de Buenos Aires (CABA), Argentina.

2 Instituto Argentino de Educación e Investigación en Nutrición - IADEIN, Ciudad Autónoma de Buenos Aires (CABA), Argentina.

\section{Summary}

The third trimester of gestation is the period of highest accretion of macro and micronutrients. Preterm newborns present a lower weight than full-term newborns. They should have a postnatal growth pace to allow them to reach a normal fetus composition of that conception age. To do so, an early nutrition should be set up to contribute several more amounts of nutrients than normally needed for full-term newborns, preventing complications during hospital stay. Nutritional deficit is difficult to compensate despite of early enteral and parenteral nutrition with fortified human milk. Iron plays an essential role and its depletion generates iron deficiency anemia which is common among this population as well as neurodevelopmental disorders. There are preventive measures such as delayed cord clamping and blood extraction control during hospitalization. However, there are some controversial issues in relation to iron doses, treatment duration, administration ways, transfusion criteria, the association with enterocolitis. In order the risk of angiogenic effect in the increase incidence of retinopathy on preterm infants. In current practice, the pediatrician faces the dilemma of deciding among different strategies to administer iron amounts so as to avoid iron deficiency anemia and also the excess related problems, so as to get rid of economic and social costs on the short and long terms, enhancing the development and health on childhood, teenage and adulthood.

Keywords: Iron; Preterm birth infant; Nutrition; Iron deficiency; Anemia.

4 Cátedra de Nutrición, Facultad de Farmacia y Bioquímica, Universidad de Buenos Aires, Ciudad Autónoma de Buenos Aires (CABA). Argentina. 


\section{INTRODUCCIÓN}

En Argentina nacen alrededor de 750.000 niños por año, de los cuales los menores de $2.500 \mathrm{~g}$ representan un 7,4 $\%$ del total de nacidos vivos y los menores de $1.500 \mathrm{~g}$ son $1,1 \%$ del total de nacimientos. En valores absolutos son más de 8.000 recién nacidos que requieren una atención especial en relación a la nutrición, con un soporte nutricional inmediato, constituyendo una emergencia nutricional $^{(1)}$.

Es de destacar que los menores de 32 semanas de gestación pueden presentar, con frecuencia, compromiso del neurodesarrollo (5\% a $15 \%$ ), con casos de parálisis cerebral y alguna alteración cognitiva, conductual, social y bajo rendimiento escolar. La nutrición adquirió importancia en los últimos años ya que es una variable modificable y tiene impacto en las diferentes morbilidades de esta población, con repercusión a corto y largo plazo. Por lo tanto, el adecuado soporte nutricional no solo se debe focalizar en el crecimiento pondoestatural, sino en el del perímetro cefálico.

El desarrollo fetal durante el embarazo, el primer y segundo año de vida son períodos críticos constituyendo lo que actualmente se conoce como "los primeros 1.000 días" durante los cuales una nutrición adecuada desde la concepción es fundamental para el desarrollo madurativo de órganos y la óptima función fisiológica posnatal. La distribución en el tiempo se muestra en el esquema presentado en la Tabla 1.

La incidencia materna de diabetes gestacional, obesidad, desnutrición y anemia actúan sobre el feto y dan lugar a situaciones de riesgo.

El nacimiento de un prematuro (RNPT) ocurre, generalmente, en el tercer trimestre del embarazo, etapa fetal donde se produce la principal acreción de macro y micronutrientes. La interrupción del pasaje transplacentario, la mayor demanda de nutrientes por el rápido crecimiento y los inadecuados depósitos aumentan las necesidades nutricionales del RNPT. Por lo tanto, la nutrición temprana es imprescindible para lograr una adecuada masa corporal, densidad ósea, optimizar el neurodesarrollo, favorecer la maduración y funcionalidad del sistema inmune, con impacto en la salud del adulto.
El soporte nutricional se debe realizar desde las primeras horas de vida, mediante nutrición parenteral con alto aporte de proteínas, glucosa, lípidos, vitaminas, macro y microminerales. Luego, la nutrición enteral trófica, prioritariamente con leche humana fortificada (LHF), hasta lograr un aporte completo enteral con LHF, que permita suspender la nutrición parenteral para llegar al alta con el menor déficit posible de nutrientes. Al final, la etapa postegreso implica succión, pecho, LHF y leche postalta ${ }^{(2)}$.

A pesar de tomar estas medidas nutricionales, los RNPT presentan restricción de crecimiento extrauterino o desnutrición posnatal ${ }^{(3-5)}$.

Los RNPT deberían presentar un ritmo de crecimiento posnatal elevado, que les permita alcanzar la composición de un feto normal de la misma edad gestacional. La nutrición fetal normal es la referencia para la nutrición posnatal en los niños prematuros y es de gran importancia jerarquizar el soporte nutricional, individualizado para que cada RNPT cumpla con un determinado ritmo de aumento de peso, estatura y perímetro cefálico ${ }^{(6)}$.

La restricción del crecimiento extrauterino, durante las primeras semanas de vida, es indicador de severo déficit nutricional en los RNPT, en especial en los menores de $1.500 \mathrm{~g}$, quienes presentan alteración del crecimiento a largo plazo ${ }^{(7,8)}$.

La Figura 1 muestra la correlación entre edad gestacional y peso al nacer, considerando como ideal el

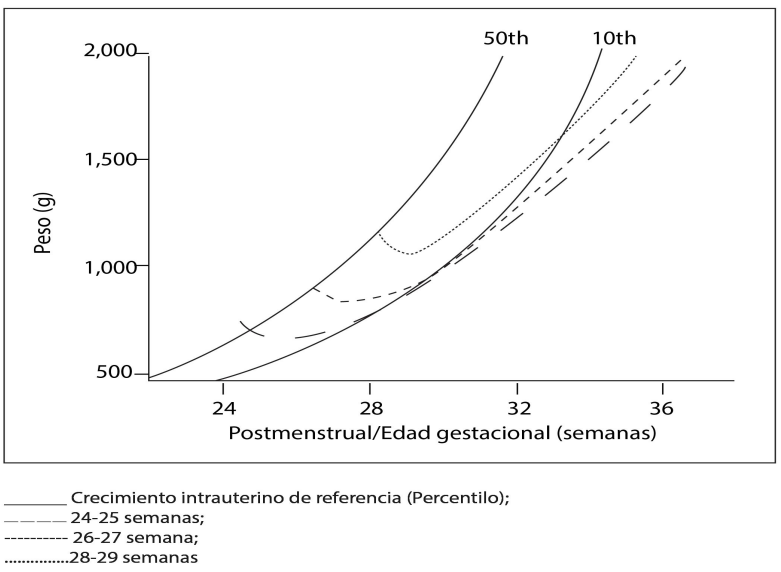

Figura 1. Restricción del crecimiento posnatal en prematuros de bajo peso al nacer.

Tabla 1. Esquema de distribución en el tiempo de los 1.000 días

\begin{tabular}{|l|l|l|}
\hline Embarazo: 270 días & Primer año: 365 días & Segundo año: 365 días \\
\hline \multicolumn{2}{|c|}{ Períodos críticos: total 1.000 días } \\
\hline
\end{tabular}


crecimiento intrauterino entre los percentiles 10 y 50 . Se observa que un prematuro que nace con menos de 29 semanas presenta disminución de peso durante los primeros días de internación.

En la unidad de cuidados intensivos (UCIN) es difícil alcanzar los aportes calórico-proteicos ideales para lograr una tasa de crecimiento adecuada. Ese déficit nutricional hace que llegue a las 36 semanas por debajo del percentil 10 y aun después del alta, resulta difícil de revertir ${ }^{(8)}$.

En la Tabla 2 figuran los rangos de las recomendaciones de nutrientes de niños pretérmino, de peso cercano a 1.800 g, según ESPGHAN $2010^{(9)}$, expresadas por $\mathrm{Kg}$ de peso corporal y por $100 \mathrm{Kcal}$. Se debe tener en cuenta que las cifras por $\mathrm{Kg}$ de peso corporal se refieren a

Tabla 2. Recomendaciones de nutrientes para niños pretérmino, de peso cercano a $1.800 \mathrm{~g}$, según ESPGHAN 2010(10)

\begin{tabular}{|c|c|c|c|}
\hline \multirow{2}{*}{ NUTRIENTES } & \multicolumn{2}{|c|}{ ESPGHAN 2010} & \multirow{2}{*}{$\begin{array}{l}\text { Leche materna PT } \\
100 \mathrm{Kcal} / \mathrm{d}\end{array}$} \\
\hline & $\mathrm{Kg} / \mathrm{d}$ & $100 \mathrm{Kcal} / \mathrm{d}$ & \\
\hline Líquido mL & $135-200$ & & 149 \\
\hline Energía (Kcal) & $110-135$ & 100 & 100 \\
\hline Carbohidratos (g) & $11,6-13,2$ & $10,5-12$ & 9,9 \\
\hline $\begin{array}{l}\text { Proteínas }(\mathrm{g}) \\
(<1 \mathrm{~kg} \text { peso) }\end{array}$ & $4,0-4,5$ & $3,6-4,1$ & 2,1 \\
\hline $\begin{array}{l}\text { Proteínas ( } \mathrm{g}) \\
(1,0-1,8 \mathrm{~kg} \text { peso })\end{array}$ & $3,5-4,0$ & $3,2-3,6$ & \\
\hline $\begin{array}{l}\text { Lípidos (g) } \\
\text { (<40\% TCM) }\end{array}$ & $4,8-6,6$ & $4,4-6$ & 5,8 \\
\hline Ácido Linoleico $(\mathrm{mg})^{*}$ & $385-1540$ & $350-1400$ & 550 \\
\hline Ácido a-linolénico (mg) & $\begin{array}{c}>55 \\
(0,9 \% \text { de } A G)\end{array}$ & $\begin{array}{c}>50 \\
(0,9 \% \text { de AG) }\end{array}$ & \\
\hline $\mathrm{DHA}(\mathrm{mg})$ & $12-30$ & $11-27$ & \\
\hline $\mathrm{AA}(\mathrm{mg}) * *$ & $18-42$ & $16-39$ & \\
\hline \multicolumn{4}{|l|}{ MINERALES } \\
\hline Sodio (mg) & $69-115$ & $63-105$ & 37 \\
\hline Potasio (mg) & $66-132$ & $60-120$ & 85 \\
\hline Cloro $(\mathrm{mg})$ & $105-177$ & $95-161$ & 82 \\
\hline Calcio (mg) & $120-140$ & $110-130$ & 37 \\
\hline Fósforo (mg) & $60-90$ & $55-80$ & 19 \\
\hline Magnesio (mg) & $8-15$ & $7,5-13,6$ & 4,6 \\
\hline Hierro $(\mathrm{mg})$ & $2-3$ & $1,8-2,7$ & 0,18 \\
\hline Zinc $(\mathrm{mg})^{* * *}$ & $1,1-2,0$ & $1,0-1,8$ & 0,51 \\
\hline Cobre $(\mathrm{mcg})$ & $100-132$ & $90-120$ & 96 \\
\hline Manganeso (mcg) & $\leq 27,5$ & $6,3-25$ & 1 \\
\hline Selenio (mcg) & $5-10$ & $4,5-90$ & 2,2 \\
\hline lodo (mcg) & $11-55$ & $10-50$ & 16 \\
\hline Fluor (mcg) & $1,5-60$ & $1,4-55$ & \\
\hline Cromo (ng) & $30-1230$ & $27-1120$ & \\
\hline Molibdeno (mcg) & $0,3-5$ & $0,27-4,5$ & \\
\hline \multirow{2}{*}{ VITAMINAS } & \multicolumn{2}{|c|}{ ESPGHAN 2010} & Leche materna PT \\
\hline & $\mathrm{Kg} / \mathrm{d}$ & $100 \mathrm{Kcal} / \mathrm{d}$ & $100 \mathrm{Kcal} / \mathrm{d}$ \\
\hline Vitamina $A$, mcg RE (1 mcg $\approx 3,33 \mathrm{UI})$ & $400-1000$ & $360-740$ & 174,3 \\
\hline Vitamina D (mcg) & $20-25 / d$ & $2,5-8,75$ & 0,075 \\
\hline Vitamina $\mathrm{E}$ mg a-TE & $2,2-11$ & $2-10$ & 1,6 \\
\hline Vitamina K1 (mcg) & $4,4-28$ & $4-25$ & 0,3 \\
\hline B1 (Tiamina), mcg & $140-300$ & $125-275$ & 31 \\
\hline B2 (Riboflavina) (mcg) & $200-400$ & $180-365$ & 72 \\
\hline B3 (Niacina) (mcg) & $380-5500$ & $345-5000$ & 224 \\
\hline B5 Ác. Pantoténico (mg) & $0,33-2,1$ & $0,3-1,9$ & 0,269 \\
\hline B6 -Piridoxina (mcg) & $45-300$ & $41-273$ & 22 \\
\hline B10 Ácido Fólico (mcg) & $35-100$ & $32-90$ & 5 \\
\hline B12 - Cobalamina (mcg) & $0,1-0,77$ & $0,08-0,7$ & 0,07 \\
\hline Biotina (mcg) & $1,7-16,5$ & $1,5-15$ & 0,6 \\
\hline Vitamina C (mg) & $11-46$ & $10-42$ & 16 \\
\hline
\end{tabular}


aportes mínimos de energía de $110 \mathrm{Kcal} / \mathrm{Kg} / \mathrm{d}$, ya que la inadecuación energética conduce a la deficiencia clínica y bioquímica de los demás nutrientes ${ }^{(10-12)}$.

ESPGHAN 2010 no hizo recomendaciones para niños de peso inferior a $1.000 \mathrm{~g}$, debido a la falta de datos en relación a la mayoría de los nutrientes, excepto para necesidades de proteínas.

En cuanto al hierro (Fe) las recomendaciones de ESPGHAN 2010 se refieren a suplementación profiláctica por vía enteral y pueden ser mayores según las condiciones clínicas y el tratamiento del niño. Además, advierte acerca de los riesgos del exceso de $\mathrm{Fe}$.

La prevención de la anemia por deficiencia de Fe, en el niño prematuro, constituye una de las preocupaciones del equipo de salud para evitar alteraciones de la respuesta inmune, de las funciones neurológicas y la susceptibilidad a infecciones, con efectos perjudiciales a largo plazo ${ }^{(4,13)}$.

Con ese objetivo el profesional puede optar por prescribir diferentes intervenciones terapéuticas, algunas muy controvertidas.

\section{Hierro y anemia en niños prematuros}

El hierro es un micronutriente mineral esencial en todas las células y puede presentarse como ferroso o férrico $\left(\mathrm{Fe}^{2+} \Leftrightarrow \mathrm{Fe}^{3+}\right)$, con diferentes características químicas. $\mathrm{El} \mathrm{Fe}$ funcional comprende la mayor parte del Fe total corporal, formando parte del HEM (hemoglobina circulante y mioglobina muscular). Interviene mediante enzimas y sistemas enzimáticos en los metabolismos energético y oxidativo y en el desarrollo cerebral, lo cual explica la asociación de la deficiencia de Fe con alteraciones del SNC en niños ${ }^{(14)}$.

El Fe no se encuentra libre en plasma, circula unido a una proteína lábil, sintetizada en el hígado, la transferrina, que lo incorpora a las células mediante la unión a receptores específicos de membrana. Para su ingreso en la célula debe reducirse a ferroso y para su depósito como ferritina (en hígado, bazo, médula ósea y sistema retículo endotelial) debe reoxidarse nuevamente a férrico, que es movilizado cuando las demandas no cubren las necesidades de la eritropoyesis. En esas reacciones redox intervienen una ferroxidasa cobre-dependiente (ceruloplasmina), la xantino-oxidasa (molibdeno y Fe dependiente) y las vitaminas $\mathrm{C}$ y A. Por consiguiente, las deficiencias de proteínas, vitaminas $\mathrm{A}$ y $\mathrm{C}$ y otros nutrientes minerales, fundamentalmente el cobre, pueden causar anemia ferropénica resistente a la administración de $\mathrm{Fe}^{(15)}$.
El déficit de Fe tiene una incidencia elevada en los prematuros, siendo los factores que lo predisponen el grado de déficit materno, los bajos depósitos por el nacimiento anticipado, el menor tiempo de vida media de los eritrocitos y la inmadurez de la eritropoyesis. Se presenta con mayor precocidad cuanto menor es la edad gestacional y el peso del RNPT. La mayor transferencia placentaria de Fe ocurre en el tercer trimestre de la gestación, que es cuando se produce el nacimiento prematuro ${ }^{(16)}$.

A partir del nacimiento los escasos depósitos se deplecionan en las primeras 6 a 8 semanas de vida, por la necesidad de realizar extracciones de sangre, el inicio de la eritropoyesis madura y la recuperación del crecimiento o "catch up".

El déficit temprano de $\mathrm{Fe}$ en el recién nacido se asocia a compromiso del neurodesarrollo, en el aspecto cognitivo y motor. En los prematuros los datos son más limitados a diferencia de los recién nacidos de término donde el compromiso cognitivo es relevante ${ }^{(9,10)}$.

El contenido de hierro del RNT es, en promedio, de $75 \mathrm{mg} / \mathrm{Kg}$, que se incorpora en su mayor parte en el tercer trimestre del embarazo, promediando 1,6 $2 \mathrm{mg} / \mathrm{Kg} /$ día $^{(17,18)}$. Además, en un parto normal hay transferencia de Fe a través de la sangre del cordón. La ligadura tardía del cordón ( 3 minutos del nacimiento) determinará un mayor hematocrito en las primeras 4 $h$, menor necesidad de transfusiones y menor riesgo de hemorragia intraventricular. Por consiguiente, la cantidad de Fe de depósito de los RNPT es variable, dependiendo de la edad gestacional y de la ligadura tardía del cordón ${ }^{(19-21)}$.

\section{Diagnóstico de anemia}

La anemia se define como "disminución de la masa de glóbulos rojos o de la concentración de hemoglobina por debajo del segundo desvío estándar, respecto de la media para edad y sexo" ${ }^{(14,22)}$.

Los indicadores clásicos de evaluación de estado nutricional con respecto al $\mathrm{Fe}$ y el diagnóstico de anemia se basan en las determinaciones de hemoglobina $(\mathrm{Hb})$, hematocrito (Hto) y recuento de glóbulos rojos, de fácil realización y rutinarias en el Laboratorio de Análisis Clínicos. Los contadores hematológicos incorporan por cálculo Volumen corpuscular medio (VCM) y concentración de hemoglobina corpuscular y concentración de $\mathrm{Hb}$ corpuscular media. En el caso del RNPT es útil tener en cuenta el recuento de reticulocitos, que permite conocer la eficacia de la eritropoyesis. 
Tabla 3. Valores promedio normales de hemoglobina $(\mathrm{g} / \mathrm{dL})$ durante los primeros 3 meses de vida según peso de nacimiento ${ }^{(28)}$

\begin{tabular}{|l|c|c|c|c|}
\hline \multicolumn{1}{|c|}{ Edad } & \multicolumn{4}{|c|}{ Peso de nacimiento } \\
\hline & $\mathbf{<} \mathbf{1 . 0 0 0} \mathbf{~ g}$ & $\mathbf{1 . 0 0 1} \mathbf{- 1 . 5 0 0 ~} \mathbf{~ g}$ & $\mathbf{1 . 5 0 1} \mathbf{- 2 . 0 0 0 ~} \mathbf{~ g}$ & $>\mathbf{2 . 0 0 0} \mathbf{~ g}$ \\
\hline Nacimiento & $16,5(13,5)$ & $16,5(13,5)$ & $16,5(13,5)$ & $16,5(13,5)$ \\
\hline 24 horas & $19,3(15,4)$ & $18,8(14,6)$ & $19,4(15,6)$ & $19,3(14,9)$ \\
\hline 2 semanas & $16,0(13,6)$ & $16,3(11,3)$ & $14,8(11,8)$ & $16,6(13,4)$ \\
\hline 1 mes & $10,0(6,8)$ & $10,9(8,7)$ & $11,5(8,2)$ & $13,9(10,0)$ \\
\hline 2 meses & $8,0(7,1)$ & $8,8(7,1)$ & $9,4(8,0)$ & $11,2(9,4)$ \\
\hline 3 meses & $8,9(7,9)$ & $9,8(8,9)$ & $10,2(9,3)$ & $11,5(9,5)$ \\
\hline
\end{tabular}

* Los valores entre paréntesis expresan el límite inferior normal (media - 2 DE).

Los valores promedio normales de $\mathrm{Hb}(\mathrm{g} / \mathrm{dL})$ durante los primeros tres meses de vida, según el peso de nacimiento figuran en la tabla $3{ }^{(23)}$.

Puede observarse que la concentración de hemoglobina en los RNPT es aproximadamente $16,5 \mathrm{~g} / \mathrm{dL}$, aun en los de muy bajo peso al nacer $(<1.000 \mathrm{~g})$. Luego existe una disminución progresiva durante el primer mes de vida extrauterina, debido a que no se ha logrado depósito de Fe en el último trimestre del embarazo y las demandas son elevadas por la expansión de la masa metabólicamente activa que implica el crecimiento.

Por otra parte, la anemia se puede deber al déficit de transferrina por deficiencia proteica; a hemodilución, por aumento del volumen plasmático; presencia de infecciones o deficiencias nutricionales que alteran la movilización y utilización del hierro ${ }^{(22)}$.

Existe disparidad de criterios en los puntos de corte de los diversos indicadores para realizar un diagnóstico preciso de deficiencia de Fe en el RNPT ${ }^{(24)}$. Además, la dosis óptima de la suplementación con Fe y la duración del tratamiento son inciertos y variables. También es necesario identificar el exceso de Fe que podría producir efectos adversos (aumento del riesgo de infecciones, exacerbación de afecciones como enterocolitis necrotizante y retinopatía del prematuro) ${ }^{(25)}$. Por lo tanto, para corroborar el diagnóstico de la anemia por deficiencia de Fe y realizar el seguimiento del paciente se aconseja determinar uno o más de los siguientes indicadores ${ }^{(26)}$ :

- Hierro sérico: los valores de referencia presentan un amplio rango. Es de utilidad para evidenciar el exceso de $\mathrm{Fe}$.

- Porcentaje de saturación de transferrina: puede evidenciar deficiencia o exceso de Fe, pero depende también de la síntesis de transferrina.

- Ferritina sérica: indica depósitos de Fe, pero valores elevados pueden deberse a procesos infecciosos o inflamatorios ${ }^{(27)}$.
- Protoporfirina eritrocitaria: aumenta cuando no hay suficiente Fe para completar la eritropoyesis, detectando deficiencias marginales de $\mathrm{Fe}^{(22)}$.

- Receptor soluble de transferrina: valores elevados reflejan bajos depósitos de Fe o incremento de la eritropoyesis, sin estar afectada por las infecciones o procesos inflamatorios, proponiéndolo como "estándar de oro" (28).

- Hepcidina: indicador promisorio, que por el momento se utiliza solamente con fines de investigación ${ }^{(29)}$.

Las determinaciones de $\mathrm{Hb}$ y de ferritina son las más aconsejadas para el diagnóstico de sobrecarga de Fe, deficiencia de Fe y anemia.

Los valores límite sugeridos para el seguimiento de lactantes con muy bajo peso al nacer $(<1.500 \mathrm{~g})$ son según la Dirección Nacional de Maternidad e Infancia del Ministerio de Salud de la Nación de la República Argentina ${ }^{(23,30)}$ se presentan en la Tabla 4.

\section{Intervenciones para prevenir la anemia ferropénica del prematuro}

Las posibles intervenciones para lograr el aporte de $\mathrm{Fe}$ similar al del tercer trimestre del embarazo son:

- Tranfusiones

- Administración de eritropoyetina

- Administración de hierro por vía parenteral

- Alimentación enteral con leche materna con agregado de Fortificadores de leche humana

- Administración de hierro por vía oral

- Fórmulas lácteas especiales para prematuros.

\section{Transfusiones}

La transfusión de glóbulos rojos (GR) es una práctica habitual en las primeras semanas de vida del RNPT y puede proporcionar 0,5 - 1,0 mg de $\mathrm{Fe} / \mathrm{mL}$ de glóbulos rojos sedimentados (GRS). La administración de cada transfusión depende del peso del niño, la edad gesta- 
Tabla 4. Valores límite sugeridos para el seguimiento de lactantes con muy bajo peso al nacer $(<1.500 \mathrm{~g})$, según la Dirección Nacional de Maternidad e Infancia del Ministerio de Salud de la Nación de la República Argentina. Adaptado de: Domellöf M. ${ }^{(30)}$.

\begin{tabular}{|l|c|c|c|c|}
\hline \multicolumn{1}{|c|}{ Indicador / edad } & Recién nacido & 2 meses & $\mathbf{4}$ meses & $\mathbf{6}$ - 24 meses \\
\hline Ferritina $(\mu \mathrm{g} / \mathrm{L})$ & & & & \\
\hline Exceso de Fe & $>300$ & $>300$ & $>250$ & $>200$ \\
\hline Deficiencia de Fe & $<35$ & $<40$ & $<20$ & $<10-12$ \\
\hline $\mathrm{Hb}(\mathrm{g} / \mathrm{dL})$ Anemia & $<13,5$ & $<9,0$ & $<10,5$ & $<10,5$ \\
\hline
\end{tabular}

cional, la función respiratoria, las patologías, los signos clínicos y los datos hematológicos ${ }^{(31)}$.

Se postula que el Fe de los glóbulos rojos de las transfusiones es utilizado lentamente para la eritropoyesis y puede producir acumulación excesiva de Fe, evidenciada por niveles elevados de ferritina y Fe sérico ${ }^{(32)}$ aconsejando controlar el hematocrito cada semana ${ }^{(33)}$.

Hsiu-Lin Chen et al ${ }^{(32)}$ estudiaron la evolución clínica y los indicadores hematológicos (reticulocitos, Fe sérico y ferritina) a los 30 días, de dos grupos de RNPT que recibieron transfusiones según un criterio "restrictivo" o "liberal". Aunque encontraron diferencias significativas en los reticulocitos, las transfusiones $>30 \mathrm{~mL}$ aumentaron el riesgo de problemas pulmonares crónicos, sugiriendo el criterio restrictivo como más adecuado.

El Consenso SIBEN 2011 ${ }^{(34)}$ recomienda transfundir 10 - $15 \mathrm{~mL} / \mathrm{Kg}$ de GRS en forma lenta $(2-4 \mathrm{~h})$.

En los RNPT la enterocolitis necrotizante (ECN) es una patología grave que se asocia a elevada morbimortalidad. Tiene una etiología multifactorial inherente a las características de los RNPT e incluso a sus antecedentes prenatales. En los últimos años se describió una asociación de transfusiones con glóbulos rojos y ECN en RNPT, aunque es difícil encontrar una causa que lo explique, se debe tomar la decisión de transfundir con criterio adecuado para evitar complicaciones ${ }^{(34)}$.

Por otro lado, la presencia de hemoglobina adulta aportada por las transfusiones constituye un factor de riesgo para la Retinopatía del Prematuro (ROP) ${ }^{(35)}$.

Controversia: la administración de GRS parece ser de utilidad para mejorar el estado nutricional respecto al Fe en el RNPR. Sin embargo, es el neonatólogo quien debe tomar una decisión acerca de esta intervención según criterios establecidos.

\section{Administración de eritropoyetina}

$\mathrm{La} \mathrm{Hb}$ fetal se sintetiza en el hígado y constituye la mayor cantidad de $\mathrm{Hb}$ en el RNPT ${ }^{(36)}$. Luego del nacimiento la
$\mathrm{Hb}$ se sintetiza en la médula ósea y, debido a la inmadurez del RNPT, para estimular la eritropoyesis normal, se suele administrar eritropoyetina, hormona sintetizada en el riñón, cuando disminuye la tensión parcial de oxígeno en la sangre y los tejidos ${ }^{(37)}$.

La administración de EPO (eritropoyetina humana recombinante) promueve la eritropoyesis, depletando los depósitos de Fe, por lo que es necesaria la suplementación con Fe. Se aconsejan dosis de 3 - $8 \mathrm{mg}$ de $\mathrm{Fe} / \mathrm{Kg}$ vía subcutánea, que son toleradas y no parecen aumentar el estrés oxidativo.

El Committee on Nutrition of the American Academy of Pediatrics recomienda suplementación enteral 6 $\mathrm{mg} / \mathrm{Kg} / \mathrm{d}$ de Fe durante la administración de EPO. Sin embargo, esas dosis pueden ser insuficientes para mantener la eritropoyesis y los depósitos de Fe.

Algunos autores indican que se reduce la necesidad de transfusiones en el RNPT, pero otros ${ }^{(36)}$ observaron que la administración temprana de EPO no redujo el requerimiento de transfusiones en la población en estudio.

Aunque otros estudios señalan beneficios, la EPO no se utiliza de rutina en las Unidades de Neonatología en nuestro país debido a que además del aumento de la eritropoiesis tiene un efecto angiogénico que aumenta la incidencia de retinopatía del prematuro. Si bien estudios recientes describen una acción neuroprotectora debido al efecto angiogénico, tanto en prematuros como en niños con encefalopatía hipóxico-isquémica, son necesarios estudios adicionales en relación a este tema para recomendar su uso rutinario ${ }^{(34)}$.

\section{Administración de hierro en RNPT que reciben nutrición parenteral (NP)}

Los recién nacidos de muy bajo peso al nacer $(<1.500 \mathrm{~g})$ reciben nutrición parenteral sin agregado de Fe intravenoso (IV) ${ }^{(38)}$.

El Fe administrado por vía intravenosa elude la absorción reguladora del intestino y se podría producir, con el tiempo, una sobrecarga de Fe. Por este motivo, se reco- 
mienda no administrar hierro IV en RNBP a menos que estén recibiendo NP a largo plazo.

En Estados Unidos el Fe no es agregado habitualmente en las mezclas de nutrición parenteral (NP) debido a su efecto sobre la la peroxidación de los lípidos que afecta la estabilidad de los demás componentes de la fórmula. Por lo tanto, los pacientes que reciben NP y presentan deficiencia de $\mathrm{Fe}$, en general lo reciben por vía enteral, en dosis variables según el estado clínico del paciente, el diagnóstico de laboratorio y el criterio médico ${ }^{(39)}$.

Sin embargo, la mayoría de los productos IV multitraza para pediatría y neonatología en Europa contienen $\mathrm{Fe}$, mientras que algunos países de Sur América (Argentina, Uruguay, Paraguay, Chile) solo disponen de productos para adultos que sí lo contienen. Por este motivo en Argentina los farmacéuticos preparan mezclas individualizadas de microminerales (sin hierro) para neonatología y pediatría.

Por otra parte, los diversos componentes individuales de macro y micronutrientes elaborados por la industria farmacéutica, que se utilizan para la preparación de las mezclas de NP pueden contener elementos minerales esenciales o tóxicos como contaminantes, no declarados en los rótulos de estos productos ${ }^{(40-42)}$. El Fe es uno de los minerales hallados en los diferentes trabajos de investigación y proviene de la contaminación no prevista y muy difícil de evitar y controlar por parte de la industria durante el proceso de fabricación ${ }^{(41)}$.

El nivel de contaminación de $\mathrm{Fe}$ en componentes utilizados en la preparación de NPT para neonatología ha resultado de mayor magnitud en las soluciones de glucosa y de lípidos ${ }^{(43)}$. Esos resultados han evidenciado que las fórmulas de NPT para neonatos de $1.200 \mathrm{~g}$ aportarían en promedio $73 \mathrm{mcg} / \mathrm{d}$ de $\mathrm{Fe}$, cantidad que no cubre los requerimientos de los prematuros. Sin embargo, estas pequeñas cantidades aportadas diariamente podrían ser incorporadas al metabolismo, pero se requieren estudios sobre el tema.

\section{Suplementación con hierro}

Es una práctica generalizada administrar suplementos de $\mathrm{Fe}$ a los neonatos prematuros y a los de bajo peso al nacer para prevenir la anemia ferropénica debido a sus escasas reservas ${ }^{(25)}$. Sin embargo, las cifras a administrar son muy controvertidas y hay grandes variaciones en las prácticas clínicas con respecto a las indicaciones, dosis, tiempo de iniciación y duración de la administración de Fe.

ESPGHAN 2010 tuvo en cuenta un metaanálisis de los estudios publicados hasta 1992 y 3 trabajos posteriores que compararon diferentes dosis de $\mathrm{Fe}^{(44-46)}$. Con base en dichos trabajos aconseja la suplementación profiláctica de $\mathrm{Fe}$ en niños pre-término de peso superior a $1.800 \mathrm{~g}$ utilizando dosis de $2 \mathrm{mg} / \mathrm{Kg} / \mathrm{d}$ de $\mathrm{Fe}$ como sulfato ferroso.

Posteriormente, una revisión sistemática de Cochrane Neonatal Group ${ }^{(25)}$ analizó 26 estudios (2.763 recién nacidos), que compararon dosis 2 vs $3 \mathrm{mg} / \mathrm{Kg} /$ día de Fe en las primeras 8 semanas y media de vida posnatal sin evidenciar beneficio hematológico al superar las dosis consideradas "estándar".

Las recomendaciones de la Sociedad Argentina de pediatría (SAP) y de la Sociedad Argentina de Hematología (SAH) son similares: para los RNPT > $1.500 \mathrm{~g}$, pero también hace recomendaciones de dosis mayores para los de peso $<1.500 \mathrm{~g}$ (Tabla 5).

PrevInfad y la American Association of Pediatrics recomiendan también una ingesta mínima de hierro de 2 $\mathrm{mg} / \mathrm{kg} /$ día. El preparado de elección que se aconseja utilizar es el sulfato ferroso. En todos los casos, la administración deberá prolongarse hasta los $12-18$ meses de edad ${ }^{(46)}$.

Tabla 5. Recomendaciones de dosis de Fe vía enteral de la Sociedad Argentina de Pediatría (SAP) y de la Sociedad Argentina de Hematología (SAH) ${ }^{(23)}$

\begin{tabular}{|l|l|l|l|}
\hline & \multicolumn{3}{|c|}{ Dosis de Fe vía enteral (mg/Kg/d) } \\
\hline $\begin{array}{c}\text { Peso al } \\
\text { nacimiento (g) }\end{array}$ & \multicolumn{1}{|c|}{$<\mathbf{7 5 0}$} & \multicolumn{1}{|c|}{$\mathbf{7 5 0}-\mathbf{1 . 5 0 0}$} & $\mathbf{1 . 5 0 0 ~ a ~ 2 . 5 0 0}$ \\
\hline & $5-6 \mathrm{mg} / \mathrm{Kg} / \mathrm{d}$ & $3-4 \mathrm{mg} / \mathrm{Kg} / \mathrm{d}$ & $2 \mathrm{mg} / \mathrm{Kg} / \mathrm{d}$ \\
$\begin{array}{l}\text { SAH } \\
\text { SAP }\end{array}$ & $\begin{array}{l}\text { Comenzando } \\
\text { durante el primer } \\
\text { mes de vida }\end{array}$ & $\begin{array}{l}\text { Durante el primer mes } \\
\text { de vida }\end{array}$ & $\begin{array}{l}\text { Antes del segundo } \\
\text { mes de vida }\end{array}$ \\
\hline
\end{tabular}


En conclusión, suplementar Fe $(2 \mathrm{mg} / \mathrm{Kg} /$ día $)$ desde las 6 semanas a los 6 meses reduce este riesgo de manera efectiva, sin efectos adversos a corto plazo sobre la morbilidad o el crecimiento.

La suplementación profiláctica de Fe por vía enteral, ya sea como suplemento o incorporado en fórmulas o en complementos de la leche humana podría comenzar a las 2 - 4 semanas de edad en niños pretérmino. Los niños que reciben eritropoyetina y aquellos que tienen pérdidas no compensadas requieren mayor dosis como suplemento.

Se acepta que dosis de Fe enteral mayores a $5 \mathrm{mg} /$ $\mathrm{Kg}$ deben evitarse en niños pretérmino debido al riesgo de posible retinopatía asociada a prematurez. La suplementación con Fe debe ser evitada si los niños reciben múltiples transfusiones y tienen elevados valores de ferritina ${ }^{(34)}$.

Controversia: los datos disponibles sugieren que los RNPT que reciben suplemento de Fe tienen un nivel de hemoglobina ligeramente mayor y un menor riesgo de desarrollar anemia por deficiencia de $\mathrm{Fe}$ en comparación con aquellos que no están suplementados. Sin embargo, el momento óptimo de inicio de la suplementación con Fe y su duración siguen sin estar definidos claramente.

Por otra parte, no queda claro si los suplementos de $\mathrm{Fe}$ en neonatos prematuros y de bajo peso al nacer tienen beneficios a largo plazo en términos de desarrollo y crecimiento del neurodesarrollo.

\section{Alimentación con leche materna y fortificadores de leche humana}

La leche materna constituye el alimento ideal para el adecuado crecimiento, desarrollo y salud del niño nacido a término. Su contenido en $\mathrm{Fe}$ es muy bajo, pero por estar unido a la lactoferrina presenta biodisponibilidad elevada (40\% - $60 \%)$. La inmadurez del recién nacido protege a la lactoferrina de la hidrólisis gástrica y permite que se transfiera el $\mathrm{Fe}$ a los receptores específicos de la mucosa intestinal, por lo cual, junto con el Fe de depósito, cubre las necesidades durante los 4 - 6 primeros meses de vida en el nacido a término.

Sin embargo, los RNPT alimentados exclusivamente con leche humana presentan deficiencias nutricionales durante y más allá del período de internación e inadecuada tasa de crecimiento ${ }^{(8)}$. El contenido de los principales nutrientes en la leche materna con relación a las recomendaciones de ESPGHAN para los $\mathrm{RNPT}^{(9)}$ se representa en la Figura 2.

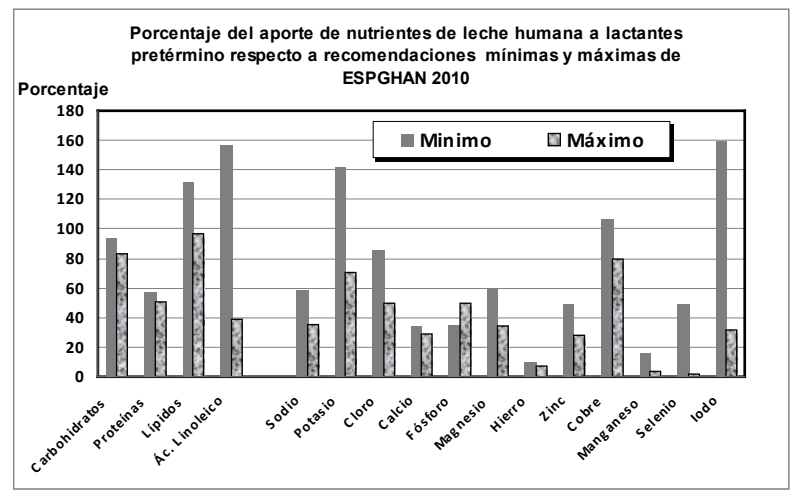

Figura 2. Porcentaje del aporte de nutrientes de leche humana a lactantes pretérmino respecto a recomendaciones mínimas y máximas de ESPGHAN 2010.

Tomado de: Pita Martín de Portela, ML y Vecchiarelli, C. Los complementos de la leche materna en la nutrición del niño prematuro.

Pese a ello, la leche de la propia madre se debe incorporar lo antes posible en el RNPT por sus múltiples beneficios: nutricionales, inmunológicos, neuromadurativos y psicofísicos, que fortalecen el vínculo madre -hijo - familia. En consecuencia, se debe recurrir a la fortificación de la leche humana para aumentar el aporte calórico y el de macro y micronutrientes ${ }^{(10)}$.

Los fortificadores de leche humana son un suplemento de multicomponentes que mejoran a corto plazo el crecimiento en ganancia de peso, estatura y perímetro cefálico, e incrementan el contenido mineral y el balance nitrogenado, sin observar efectos adversos a corto y largo plazo. Se incorporan cuando el aporte de leche materna es entre 50 a $100 \mathrm{~mL} / \mathrm{Kg} /$ día ${ }^{(49)}$. La incorporación de multicomponentes, a pesar de establecer mínimos cambios en la osmolaridad, el vaciamiento gástrico y algunas propiedades inmunológicas, parece ser la mejor opción para mejorar el crecimiento.

En la actualidad, la industria farmacéutica ofrece varias opciones de fortificadores, con algunas variaciones entre ellos, pero existen discrepancias en cuanto al agregado de $\mathrm{Fe}$ a los complementos de leche materna. Por lo tanto, en la práctica el pediatra puede enfrentarse con la disyuntiva de elegir entre fórmulas comerciales disponibles, teniendo en cuenta las características clínicas del RNPT.

\section{Fórmulas especiales y fórmulas lácteas para prematuros}

La investigación de las últimas décadas ha permitido el diseño de fórmulas especiales para prematuros que 
deben usarse durante el período intrahospitalario según las características de cada prematuro y su evolución hasta los 3, 6 y 9 meses de edad corregida.

Si bien no se han comprobado ventajas estadísticamente significativas entre la leche humana fortificada y la leche de fórmula para prematuros en relación con la ganancia de peso, se debe jerarquizar la posibilidad de aportar leche humana por otras ventajas: presenta una alta biodisponibilidad del $\mathrm{Fe}$, una relación calcio/fósforo (2:1), que favorece la absorción de estos minerales; realiza un adecuado aporte de ácidos grasos de cadena larga, de gran importancia para el desarrollo cerebral, que favorecen el neurodesarrollo ${ }^{(4)}$.

En caso de no lograr el suficiente almacenamiento de leche humana o si los RNPT no pueden ser alimentados con leche materna luego del alta se recurrirá a leche de fórmula especialmente diseñada para los prematuros que pueden contener entre 8 y $14,6 \mathrm{mg}$ de hierro/L. Sin embargo, pese a recibir cerca de 1,5 a 2,2 mg/Kg/día de $\mathrm{Fe}$ con la fórmula, se estima que $14 \%$ de los recién nacidos prematuros desarrollarán ferropenia entre los 4 y 8 meses de edad.

\section{Biodisponibilidad del hierro por vía enteral y tipos de sales utilizadas para la suplementación oral de Fe en prematuros}

La biodisponibilidad de un nutriente se define como "la proporción del nutriente ingerido que puede ser absorbido y utilizado por el organismo para los fines que le son propios".

La absorción del Fe tiene lugar en la porción superior del intestino delgado, para lo cual debe encontrarse en forma soluble al $\mathrm{pH}$ del tracto gastrointestinal. Los iones ferrosos permanecen solubles hasta un $\mathrm{pH} \cong 7$, mientras que los férricos forman, a $\mathrm{pH}>3$, hidróxidos hidratados altamente insolubles. Los distintos componentes del producto o los producidos durante la digestión, pueden formar complejos cuya solubilidad varía según su naturaleza química y el $\mathrm{pH}$. Los complejos solubles incrementan la biodisponibilidad (biodisponibilidad: algunos amino ácidos, hidratos de carbono, ácidos orgánicos (como málico, cítrico, láctico) y vitamina $\mathrm{C}$, que es el potenciador más efectivo. Por otra parte, pueden formarse complejos insolubles que reducen la absorción (fosfatos, fitatos, polifenoles, fibra, ácidos grasos, algunas proteínas, etc.). Existen además, diversas interacciones con otros minerales y con vitaminas.

El sulfato ferroso suele tomarse como referencia al determinar la absorción del Fe. Su absorción en solución acuosa, medida isotópicamente en individuos adultos depletados, es de $40 \%$. Las formas divalentes de hierro $\left(\mathrm{Fe}^{2+}\right)$ son las más utilizadas para la suplementación en bebés prematuros, pero a menudo conducen a síntomas gastrointestinales como vómito, cólicos y estreñimiento.

Los diversos compuestos de Fe utilizados en la fortificación exhiben una dicotomía entre su absorción y su reactividad: los de mayor biodisponibilidad poseen una mayor reactividad, es decir un mayor efecto prooxidante y, por ende, menor compatibilidad con el vehículo. A la inversa, los compuestos de menor biodisponibilidad poseen mayor compatibilidad desde el punto de vista de las características nutritivas del producto. Existen como excepciones complejos que por su elevada constante de estabilidad se absorben sin disociar y presentan alta biodisponibilidad sin producir interacciones indeseables con la matriz en la que se incorporan.

El hierro trivalente $\left(\mathrm{Fe}^{3+}\right)$ complejado con polisacáridos de bajo peso molecular fue comparado con el sulfato ferroso, sin diferencias significativas en los dos grupos y la suplementación fue bien tolerada. Por lo tanto, en el lactante prematuro el uso de complejos de Fe trivalentes puede ser considerado como una buena alternativa ${ }^{(45)}$.

La fortificación debe asegurar que el nutriente agregado sea fisiológicamente disponible en el vehículo, estable en las condiciones adecuadas de almacenamiento y uso del producto fortificado, que no produzca desequilibrios de nutrientes esenciales y que exista una seguridad razonable de que no ocurra una ingesta excesiva a nivel de efectos adversos o toxicidad. El agregado de Fe a la leche materna mediante un fortificador debe tener en cuenta estas interacciones y evitar efectos no deseados tanto en la estabilidad del producto como en los efectos adversos en el organismo. Otros compuestos de Fe utilizados han sido Fe coloidal, Fe trivalente como complejo hidróxido polimaltosato y succinato ferroso ${ }^{(47,48)}$.

\section{Efectos adversos del agregado de hierro}

En contraste con otros nutrientes no hay un mecanismo de excreción que proteja del exceso de $\mathrm{Fe}$, que es un potente prooxidante y cuyo exceso puede producir aumento del riesgo de infección, disminución del crecimiento y de la absorción de otros minerales esenciales como el $\mathrm{Zn}{ }^{(49)}$.

El exceso de Fe puede permitir un mayor crecimiento de los microorganismos y un proceso más virulento. En el intestino puede unirse a la lactoferrina y disminuir la función protectora de esta proteína sobre los microorganismos patógenos. Ovali y col evidenciaron que el 
agregado de $\mathrm{Fe}$ a leche materna con fortificador reduce la capacidad antimicrobiana frente a la E. coli, S. aureus $y$ P. aeruginosa. Este efecto puede deberse a disminución del efecto de la lactoferrina presente naturalmente en la leche materna por su saturación con el Fe agregado ${ }^{(49)}$.

Además, cuando existe sobrecarga de $\mathrm{Fe}$, se excede la capacidad de saturación de la transferrina y el Fe libre promueve la formación de radicales libres aumentando el riesgo de retinopatía asociada a prematurez, y de radicales libres que pueden inducir peroxidación lipídica.

Las dosis de Fe enteral mayores a $5 \mathrm{mg} / \mathrm{Kg}$ deben evitarse en niños pretérmino debido al riesgo de posible retinopatía asociada a prematurez. La suplementación con $\mathrm{Fe}$ debe ser evitada si los niños reciben múltiples transfusiones y tienen elevados valores de ferritina.

$\mathrm{El} \mathrm{Fe}$ es un potente catalizador de reacciones que generan radicales libres que, en presencia de oxígeno molecular, inician la peroxidación en cadena sobre los dobles enlaces de los ácidos grasos poliinsaturados de las membranas celulares. Esto dejará una membrana disfuncional y una posible muerte celular.

Estas reacciones también pueden producirse en las formulaciones disminuyendo la vida útil de los productos y la biodisponibilidad de otros nutrientes. Por lo tanto, en dichas formulaciones el agregado de Fe debe ser cuidadosamente analizado en cuanto a su interacción con los otros componentes ${ }^{(49)}$.

\section{CONCLUSIONES Y CONTROVERSIAS}

- Durante las primeras semanas de vida del recién nacido prematuro es común la restricción del crecimiento extrauterino debido al severo déficit nutricional, que repercute en el crecimiento a largo plazo.

- El soporte nutricional se debe realizar desde las primeras horas de vida para evitar la desnutrición posnatal. Desde las primeras horas el profesional debe optar por prescribir diferentes intervenciones terapéuticas, algunas muy controvertidas.

- La prevención de la anemia por deficiencia de Fe en el niño prematuro constituye una de las decisiones a tomar por el equipo de salud para prevenir efectos perjudiciales a largo plazo.

- La ligadura tardía del cordón umbilical es una de las primeras precauciones para lograr el aumento del hematocrito y reducir el número de transfusiones.

- Para aportar cantidades de Fe similares al tercer trimestre del embarazo y prevenir la anemia ferropénica del prematuro se pueden administrar transfusiones de sangre, eritropoyetina, hierro por vía parenteral o por vía oral, alimentación enteral con leche materna fortificada y fórmulas lácteas especiales para prematuros. Es el neonatólogo quien debe tomar una decisión acerca de estas intervenciones según criterios establecidos y el estado clínico del niño.

- La nutrición parenteral es el primer alimento que recibe el RNPT. Se ha demostrado que existe contaminación con Fe en los componentes utilizados en su elaboración. Las mezclas de NP para neonatología contienen pequeñas cantidades de Fe que aportadas diariamente podrían ser incorporadas al metabolismo, pero se requieren estudios sobre el tema.

- Existe disparidad de criterios en los puntos de corte de los diversos indicadores para realizar un diagnóstico preciso de deficiencia de Fe y el seguimiento del RNPT, en la dosis óptima de la suplementación y la duración del tratamiento.

- Se recomiendan suplementos de Fe de $2 \mathrm{mg} / \mathrm{Kg} /$ día desde 6 semanas a 6 meses para reducir el riesgo de manera efectiva, sin efectos adversos a corto plazo sobre la morbilidad o el crecimiento. Sin embargo, el momento óptimo de inicio de la suplementación con hierro y su duración siguen sin estar definidos claramente.

- Las dosis de Fe enteral mayores a $5 \mathrm{mg} / \mathrm{Kg}$ deben evitarse en niños pretérmino debido a la retinopatía asociada a prematurez. La suplementación con Fe debe ser evitada si los niños reciben múltiples transfusiones $y$ tienen elevados valores de ferritina

- La leche materna tiene muy bajo contenido de Fe y pese a su elevada biodisponibilidad no cubre las necesidades del nacido pretérmino. Los RNPT alimentados exclusivamente con leche humana presentan deficiencias nutricionales incluyendo la de hierro, por ello es necesario recurrir a fortificadores o a uso de suplementos orales que permiten dosificar la cantidad a administrar y evitar los efectos adversos del exceso.

- El sulfato ferroso suele tomarse como referencia y el uso de complejos de Fe trivalentes puede ser considerado como una buena alternativa.

- $\quad$ El Fe es un potente catalizador de reacciones que generan radicales libres y en presencia de oxígeno molecular inician la peroxidación en cadena.

- Estas reacciones también pueden producirse en las formulaciones disminuyendo la vida útil de los productos y la biodisponibilidad de otros nutrientes. 
Por lo tanto, en dichas formulaciones el agregado de Fe debe ser cuidadosamente analizado en cuanto a su interacción con los otros componentes.

\section{Conflicto de intereses}

No declara.

\section{Referencias bibliográficas}

1. Salud Materno Infanto-juvenil en cifras. UNICEF-Sociedad Argentina de Pediatría (SAP). Ciudad Autónoma de Buenos Aires. Argentina; 2015. Consultado febrero 2018: Disponible en: www.unicef.org/argentina

2. Adamkin DH. Feeding in preterm infant. In: Perinatal Nutrition Optimizing Infant Health and Developmental. J Bathis, Ed. New York: Marcel Dekker, 2004; 165-90.

3. Neu J, Hauser N. Postnatal nutrition and adult heath programming. Semin Fetal Neonatal Med. 2007;12(1): 78-86.

4. Toca M, Tonietti M, Vecchiarelli C. Nutrición pre y postnatal: Impacto a largo plazo en la salud. Arch Argent Pediatr. 2015;113(3):248-53.

5. Nutrición del Niño Prematuro. Recomendaciones para las Unidades de Cuidado Intensivo Neonatal. Dirección Nacional de Maternidad e Infancia. Ministerio de Salud. Presidencia de la Nación. Edición 2015; p. 2-48.

6. De Curtis M, Rigo J. Extrauterine growth retardation in verylow-birth weigth infants. Acta Paediatr. 2004; 93(12):1563-8.

7. Coverston CR, Schwartz R. Extrauterine growth restriction: a continuing problem in the NICU. Am J Matern Child Nurs. 2005; 30(2):101-6.

8. Ziegler EE. Meeting the Nutritional Needs of the Low-BirthWeight Infant. Ann Nutr Metab. 2005; 58 (suppl. 1):8-18.

9. Agostoni C, Buonocore G, Carnielli VP, De Curtis M, Darmaun D, Decsi T, et al. Enteral nutrient supply for preterm infants: commentary from the European Society of Paediatric Gastroenterology, Hepatology and Nutrition Committee on Nutrition. J Pediatr Gastroenterol Nutr. 2010; 50:85-91.

10. European Society of Paediatric Gastroenterology and Nutrition. Committee on Nutrition of the Preterm Infant Nutrition and feeding of preterm infants. Acta Paediatr Scand Suppl. 1987;336:1-14.

11. Embleton NE, Pang N, Cooke Rj. Postnatal malnutrition and growth retardation: an inevitable consequence of current recommendation in preterm infants? Pediatrics. 2001;107:270-3.

12. Ronnholm KAR, Siimes MA. Haemoglobin concentration depends on protein intake in small preterm infants fed human milk. Archives of Disease in Childhood; 1985;60:99-104.

13. Dallman PR. Iron deficiency and the immune response. Am J Clin Nutr. 1987; 46:329-34.

14. Baker R, Greer F and The Committee on Nutrition. Diagnosis and prevention of iron deficiency and iron-deficiency anemia in infants and young children ( 0 - 3 years of age). Pediatrics. 2010;126:1040-50.
15. Jian Wang, Kostas Pantopoulos. Regulation of cellular iron metabolism. Biochem. J. 2011; 434:365-81.

16. Langini SH, Portela ML, Lázzari A, Ortega Soler CR, Lönnerdal B. Do indicators of maternal iron status reflect placental iron status at delivery? J Trace Elem Med Biol. 2006; 19: 243-9.

17. Rao R, Georgieff M. Iron Therapy for Preterm Infants. Clin Perinatol. 2009; 36(1):27-42.

18. Domellof M, Braegger C, Campoy C, Colomb V, Decsi T, Fewtrell M, et al. Iron Requirements of Infants and Toddlers. On Behalf of the ESPGHAN Committee on Nutrition. JPGN. 2014;58:119-29.

19. Ceriani Cernadas JM, Carroli G, Pellegrini L, Ferreira M, Ricci C y col. Efecto del clampeo demorado del cordón umbilical en la ferritina sérica a los seis meses de vida. Estudio clínico controlado aleatorizado. Arch Argent Pediatr. 2010;108 (3):201-8.

20. Rabe H, Reynolds G, Diaz-Rossello J. Clampeo precoz versus clampeo tardío del cordón umbilical en prematuros (Revisión Cochrane traducida). En: La Biblioteca Cochrane Plus, número 4, 2007. Oxford, Update Software Ltd. Disponible en: http:// www.update-software.com (Traducida de The Cochrane Library, 2007 Issue 4. Chichester, UK: John Wiley \& Sons, Ltd.).

21. Tarnow-Mordi W, Morris J, Kirby A, Robledo K, Askie L, Brown R, et al. Delayed versus inmediate Cord Clamping in Preterm Infants. N Engl J Med. 2017;377:2445-55. Disponible en: http://www.maternoinfantil.org/archivos/ A53.PDF. Consultado en febrero de 2018.

22. Gibson RS. Assessment of Iron Status. In: Gibson RS, editor. Principles of Nutritional Assessment. New York - Oxford. Oxford University Press; 1990; p. 349-76.

23. Comité Nacional de Hematología, Oncología y Medicina Transfusional, Comité Nacional de Nutrición. Deficiencia de hierro y anemia ferropénica. Guía para su prevención, diagnóstico y tratamiento. Arch Argent Pediatr. 2017;115(Supl 4):68-82.

24. Cerami C. Iron Nutriture of the Fetus, Neonate, Infant and Child. Ann Nutr Metab. 2017; 71(suppl 3):8-14.

25. Mills RJ, Davies MW. Enteral iron supplementation in preterm and low birth weight infants. Cochrane Library. Cochrane Database of Systematic Reviews, Issue 3. Art. No.: CD005095. 2012:1-84.

26. Lafay M. Exploración de una anemia. Acta Bioquím Clín Latinoam. 2003;37 (2):203-28.

27. Langini SH, Fleischman S, López LB, Moratal Ibáñez L, Lardo MM, Ortega Soler CR, Pita Martín de Portela ML. Utilidad de la ferritina sérica para evaluar depósitos de hierro maternos en el post parto inmediato. Acta Bioquímica Clínica Latinoamericana. 2004;38(2):173-9.

28. Cook JD, Skikne BS, and Baynes RD. Serum Transferrin Receptor. Ann. Rev. Med. 1993; 44: 63-74. 
29. Collins JF, Wessling-Resnick M and Knutson MD. Hepcidin regulation of Iron transport. J. Nutr. 2008;138: 2284.

30. Domellöf M. Nutritional care of premature infants: microminerals. World Rev Nutr Diet. 2014;110:300-5.

31. Madhava R Beeram, MD, David R. Krauss, MD, Mark W. Riggs. Red blood cell transfusion practices in very low birth weight infants in 1990s postsurfactant era. J Natl Med Assoc. 2001;93:405-9.

32. Hsiu-Lin Chen, Hsing-I Tseng, Chu-Chong Lu, San-Nan Yang, Hui-Chen Fan, Rei-Cheng Yang. Effect of Blood Transfusions on the Outcome of Very Low Body Weight Preterm Infants under Two Different Transfusion Criteria Pediatr Neonatol. 2009;50(3):110-6.

33. Raghavendra Rao. Georgieff MK. Iron Therapy for Preterm Infants. Clin Perinatol. 2009; 36(1):27- 42.

34. Golombek SG, Fariña D, Sola A, Baquero H, Cabañas F, Dominguez F y col. Segundo Consenso Clínico de la Sociedad Iberoamericana de Neonatología (SIBEN). Manejo hemodinámico del recién nacido. Rev Panam Salud Pública. 2011;29(4):281-302.

35. Guía de Práctica Clínica para la prevención, diagnóstico y tratamiento de la Retinopatía del Prematuro (ROP). Dirección Nacional de Maternidad, Infancia y Adolescencia. Ministerio de Salud de la Nación. Edición 2016. Buenos Aires, Argentina.

36. Donato H, Vain N, Rendo P, Vivas N, Prudent L, Larguía M, Digregorio J, Vecchiarelli C, et al. Effect of Early Versus Late Administration of Human Recombinant Erythropoietin on Transfusion Requirements in Premature Infants: Results of a Randomized, Placebo-Controlled, Multicenter Trial. Pediatrics. 2000;105:1066-72.

37. Linxia Qiao, Qingya Tang, Wenying Zhu, Haiyan Zhang, Yuefang Zhu, Hua Wang. Effects of early parenteral iron combined erythropoietin in preterm infants. A randomized controlled trial. Medicine. 2017; 96:9(e5795).

38. Friel JK, Andrews WA, Hall MS, Rodway MS, Keith M, McCloy $\mathrm{CU}$, et al. Intravenous Iron Administration to Very-Low-Birth-Weight Newborns Receiving Total and Partial Parenteral Nutrition. JPEN J Parenter Enteral Nutr. 1995;19(2):114-8.

39. Fallon EM, Nehra D, Potemkin AK, Gura KM, Simpser E, Compher C. A.S.P.E.N. Clinical guidelines: nutrition support of neonatal patients at risk for necrotizing enterocolitis. JPEN J Parenter Enteral Nutr. 2012;36(5):506-23.
40. Pluhator-Murton MM, Fedorak RN, Audette RJ, Marriage BJ, Yatscoff RW, Gramlich LM. Trace element contamination of Total Parenteral Nutrition. 1- Contribution of Component Solutions. JPEN J Parenter Enteral Nutr. 1999;23(4): 222-7.

41. Menéndez AM, Weisstaub AR, Montemerlo H, Rusi F, Guidoni M, Piñeiro A, Pita Martín de Portela ML. Contenido de zinc y cobre en los componentes individuales de las mezclas para fórmulas pediátricas de nutrición parenteral total. Nutrición Hospitalaria. 2007;22(5):545-51.

42. Menéndez AM, Farías SS, Servant R, Morisio Y, Misischia Y, Simon S, Pita Martín de Portela ML. Contenido de aluminio en componentes individuales utilizados para preparar mezclas de nutrición parenteral en Argentina y su comparación con la legislación internacional. Nutrición Hospitalaria. 2014;29(6):1380-7.

43. Menéndez AM, Farías SS, Servant R, Cortez I, Montemerlo HJ, Weisstaub A R, Russi F, Pita Martin de Portela ML. Iron contamination in parenteral nutrition mixtures. Ann Nutr Metab. 2017;71(suppl 2):425.

44. Franz AR, Mihatsch WA, Sander S, Kron M, Pohlandt F. Prospective randomized trial of early versus late enteral iron supplementation in infants with a birth weight of less than 1301 grams. Pediatrics. 2000;106:700-6.

45. Friel JK, Andrews WL, Aziz K, Kwa PG, Lepage G, L’Abbe MR. A randomized trial of two levels of iron supplementation and developmental outcome in low birth weight infants. J Pediatr. 2001;139:254-60.

46. Sánchez Ruiz-Cabello FJ. Prevención primaria y cribado de ferropenia en lactantes. En: Recomendaciones PrevInfad / PAPPS [en línea]; 2011. Consultado: febrero 2017 [Disponible en http://www.aepap.org/previnfad/ferropenia.htm

47. Hall RT, Wheeler RE, Benson J, Harris G, Rippetoe L. Feeding iron-fortified premature formula during initial hospitalization to infants less than 1800 grams birth weight. Pediatrics. 1993;92(3):409-14.

48. Hong-Xing Jin, Rong-Shan Wang, Shu-Jun Chen, Ai-Ping Wang, Xi-Yong Liu. Early and late Iron supplementation for low birth weight infants: a meta-analysis Cochrane Neonatal Group Jin et al. Italian Journal of Pediatrics. 2015;41:16.

49. Pita Martín de Portela, María Luz y Vecchiarelli, Carmen. Los complementos de la leche materna en la Nutrición del niño prematuro. Rev Farmacéutica. 2012;54:18-38. 